\title{
Ascitis Secondary to Budd Chiari Syndrome Managed By Endovascular Stenting
}

\author{
Authors \\ Dr Hasmukh B Vora, Dr Sushruth Shetty, Dr Mahendra S Bhavsar, \\ Dr Premal R Desai, Dr Nikhil Jillawar \\ V.S. General Hospital, N.H.L.M.C, Ahmadabad, Gujarat
}

\section{Introduction}

Budd-Chiari syndrome (BCS) is an uncommon condition characterized by obstruction of the hepatic venous outflow. Presentation varies from a completely asymptomatic condition to fulminant liver failure ${ }^{1 .}$ BCS is an example of post sinusoidal portal hypertension. The management can be divided into three main categories: medical, surgical, and endovascular ${ }^{2}$. The purpose of this article is to present a case of BCS with ascites managed by endovascular stenting.

\section{Case report}

A 40 year old male presented with ascites since one year. Ascites was gradual in onset and progressive. Patient was initially treated with diuretics which showed only partial response. His ascites was transudative in nature. Patient was also found to have an elevated RBC indices in the form of $\mathrm{Hb}$ of 18 and haematocritt of $60 \%$. His WBC and platelet counts were within normal limits. Patient was diagnosed to have polycythemiavera and was started on treatment for the same. Patient underwent magnetic resonance portovenogram which showed thrombus in middle hepatic vein and doubtful occlusion of the intrahepatic IVC.
Invasive venogram was performed and pressure gradients studied. It showed a near total occlusion of the MHV and a patent IVC. Self-expanding metallic stent was placed across the MHV thrombus and good flow was demonstrated post procedure.

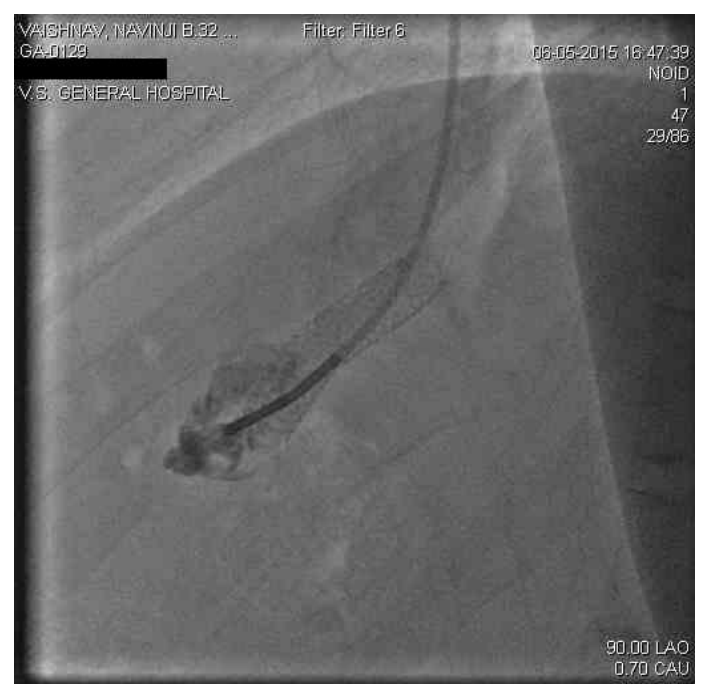

Following stenting patient had decrease in his abdominal girth and resolution of ascites in three week time. Patient was then withdrawn of diuretics and remained asymptomatic during 6 months follow up period by weekly weight charting and monthly USG abdomen. Follow up Doppler shows a normal patency of the stent. 


\section{Discussion}

BCS is a complex disorder characterized by hepatic venous outflow obstruction that may be located from small hepatic veins to the atriocaval junction $^{3 .}$ Patients with BCS may present with hepatomegaly, right upper quadrant pain, ascites, or be asymptomatic ${ }^{4}$. Percutaneous endovascular intervention has been increasing in the treatment of BCS in recent years. Restoration of good outflow in one of the three main hepatic veins is usually adequate to resolve BCS symptoms ${ }^{5}$. In BCS, both endovascular and surgical interventions provide excellent results and potentially halt liver parenchymal deterioration caused by portal hypertension. Liver transplantation remains the ultimate solution for advanced liver failure.

\section{Conclusion}

Endovascular management of ascites secondary to Budd chiari syndrome in the form of stenting is effective in the short term treatment of segmental thrombosis and leads to resolution of ascites.

\section{References}

1. Aydinli M, Bayraktar Y. Budd-Chiari syndrome: etiology, pathogenesis and diagnosis. World J Gastroenterol 2007; 13:2693-2696.

2. Plessier A, Valla DC. Budd-Chiari syndrome. Semin Liver Dis 2008; 28:259-269.

3. Hoekstra, J. and Janssen, H.L.A. Vascular liver disorders (I): diagnosis, treatment and prognosis of Budd-Chiari syndrome. Netherl J Med. 2008; 66: 334-339

4. Valla, D.C. The diagnosis and management of the Budd-Chiari syndrome: consensus and controversies. Hepatology. 2003; 38: 793-803.

5. Eapen, C.E., Velissaris, D., Heydtmann, M., Gunson, B., Olliff, S., and Elias, E. Favourable medium term outcome following hepatic vein recanalisation and/or transjugular intrahepatic portosystemic shunt for Budd Chiari syndrome. Gut. 2006; 55: 878-884. 